\title{
Natura@economía
}

ISSN 2226-9479 (Versión electrónica) Website: http://revistas.lamolina.edu.pe/index.php/neu

\section{Evaluación de riesgo ambiental generado por pasivo ambiental minero en la calidad de agua superficial} Evaluation of environmental risk generated by minimum environmental liability in
the superficial water quality

\author{
Joel Jesús Cervantes Neira ${ }^{1}$; Samuel Jesús Quito Quilla²*
}

${ }^{1}$ Universidad Nacional Agraria La Molina, Lima, Perú. joel.cerv88@gmail.com; quito.sammy@gmail.com

Recepción: 03/02/2020; Aceptación: 15/05/2020

\begin{abstract}
Resumen
En el Perú se ha venido desarrollando la minería desde hace varios siglos, por ello no existía la preocupación de cerrar los componentes de una mina, ni remediar los impactos negativos generados por los Pasivos Ambientales Mineros (PAMs). Sin embargo, en la actualidad hay leyes que promueven la identificación, inventario, evaluación y remediación de los PAMs; pero el proceso de Evaluación de Riesgo Ambiental (ERA) no se ha desarrollado lo suficiente. Este trabajo de investigación tiene como objetivo estimar el nivel de riesgo ambiental para los PAMs en la calidad de agua superficial de la localidad de San Miguel de Viso, siguiendo la metodología de la Guía de Evaluación de Riesgo Ambiental propuesta por el Ministerio del Ambiente (MINAM); y que tiene la característica principal de estimar los riesgos en el entorno humano, natural y socioeconómico estableciendo indicadores medibles para cada entorno. Los resultados obtenidos para los pasivos evaluados son que la tolva de mineral (PAS-1) y la bocamina (PAS-14) representan un nivel de riesgo moderado; y la relavera (PAS-8) y la bocamina (PAS-16) representan un nivel de riesgo significativo para la calidad de agua superficial, respectivamente.
\end{abstract}

Palabras clave: pasivo; riesgo; gravedad; probabilidad; peligro.

\begin{abstract}
In Peru mining has been developing for several centuries, therefore there was no concern to close the components of a mine, or remedy the negative impacts generated by the Environmental Mining Liabilities (PAMs). However, at present there are laws that promote
\end{abstract}

Forma de citar el artículo: Cervantes, J.; Quito, S. 2020. Evaluación de riesgo ambiental generado por pasivo ambiental minero en la calidad de agua superficial. Natura@economía 5(1): 1-14 (2020). 
the identification, inventory, evaluation and remediation of PAMs, but the Environmental Risk Assessment (ERA) process has not been developed sufficiently. This research work aims to estimate the level of environmental risk for PAMs in the quality of surface water in the San Miguel de Viso locality, following the methodology of the Environmental Risk Assessment Guide proposed by the Ministry of Environment (MINAM); and that has the main characteristic of estimating the risks in the human, natural and socioeconomic environment, establishing measurable indicators for each environment. The results obtained for the liabilities evaluated are that the ore hopper (PAS-1) and the pithead (PAS-14) represent a moderate level of risk; and the tailings deposit (PAS-8) and pithead (PAS-16) represents a significant level of risk for surface water quality, respectively.

Keyword: liabilities; risk; severity; probability; danger.

\section{Introducción}

El año 2010 el Ministerio del Ambiente (MINAM) publica la Guía de Evaluación de Riesgo Ambiental, esta guía es una herramienta de apoyo para la gestión ambiental con la finalidad de determinar los niveles de riesgo en un área geográfica, afectada o propensa a sufrir daños de origen antrópico o natural, basada en indicadores medibles y criterios de evaluación establecidos a partir de los estándares de calidad ambiental de la normativa nacional vigente o por las instituciones de derecho público internacional que sean aplicables Esta guía analiza el entorno humano, que hace referencia a las personas potencialmente afectadas que residen dentro del área de influencia del área de estudio; el entorno natural, respecto a los recursos o elementos afectados que proveen vida; y el entorno socioeconómico, en referencia a la afectación a las diferentes actividades económicas o problemas socioambientales (MINAM, 2010).

En el año 2013 el MINAM mediante resolución de consejo directivo $\mathrm{N}^{\circ} 002$ 2013-OEFA/CD, se establece la metodología para la estimación del nivel de riesgo de pasivos ambientales en el subsector de hidrocarburos, la cual se toma en cuenta los lineamentos establecidos en la guía de evaluación de riesgo ambiental publicada por el MINAM (2010).

Según el Ministerio de Energía y
Minas (MINEM), los PAMs identificados aumentan cada año. El último inventario oficial realizado en el 2018 ha identificado ocho mil setecientos noventa y cuatro (8 794) pasivos mineros a nivel nacional; de los cuales el $29 \%$ se encuentran en gestión de remediación $\mathrm{y} / \mathrm{o}$ reaprovechamiento y el $19 \%$ cuenta con un plan de cierre de pasivos aprobado. Por lo tanto el $71 \%$ solo está identificado, mas no se ha realizado un análisis de riesgo ambiental que generan estos pasivos al medio ambiente, específicamente al recurso hídrico.

El año 2011 el MINAM realizó la "Evaluación de Riesgos Ambientales de Emisiones Atmosféricas y Efluentes por Actividad Minera-Metalúrgica en la Provincia de Yauli - La Oroya”, basándose en la Guía de Evaluación de Riesgos Ambientales, analizando el factor humano, ecológico y socio económico llegando a las siguientes conclusiones; disminución de la población pecuaria, disminución de las áreas agrosilvopastoriles, disminución de los ingresos económico y alto costo de vida, nivel de riesgo ambiental en el entorno humano $86,65 \%$, entorno natural $87,17 \%$ y entorno socioeconómico 80,5\%. Así mismo un nivel de riesgo ambiental del $84,77 \%$ con un nivel significativo.

Los PAMs son todas aquellas instalaciones, efluentes, emisiones, restos o depósitos de residuos producidos por operaciones mineras, abandonadas o 
inactivas (Art. 4 del D.S. Nº 059-2005-EM. Reglamento de Pasivos ambientales en la actividad minera).

Según las fichas PERCAN aprobado mediante Resolución Directoral $\mathrm{N}^{\circ}$ 173-2009-MINEM-DGM, los pasivos pueden ser según el tipo de labor minera (tajo, pique, chimenea, bocamina, tajeo comunicado, media barreta, trinchera y rampa), según residuos mineros (material de desbroce, desmonte de mina, escorias, pilas de lixiviación, relaves, residuos de carbón, lodos de neutralización y suelo orgánico), edificación infraestructura (campamentos, oficinas, talleres, caminos, helipuertos, pistas de aterrizaje, líneas férreas, líneas eléctricas, generadores, trasformadores, planta de procesamiento, chancadoras, patios de testigo de perforación diamantina), sustancias químicas almacenadas o derrame (reactivos de proceso, aceites, combustibles, solventes, explosivos, cianuro y reactivos de laboratorio) y otros residuos (residuos industriales, domésticos y/o de construcción)

Como toda actividad productiva, la minería produce impactos. El término impacto según la dirección general de calidad ambiental del MINAM, hace referencia a cualquier cambio, modificatoria o alteración de los elementos del medio ambiente o de las relaciones entre ellos, causada por una o varias acciones (proyecto, actividad o decisión).

El mayor impacto ambiental de los PAMs se da por la contaminación de las aguas superficiales y subterráneas.

La contaminación de agua se considera como tal, cuando se altera su composición de tal forma que resulta menos apta para cualquier o todas las funciones y propósitos para los que sería apropiada en su estado natural. Alteraciones tales como: propiedades físicas, químicas y biológicas, asimismo, la descarga de sustancia líquidas, gaseosas o sólidas que producirán alteraciones en las aguas, siendo un peligro para la salud pública, la ganadería, la agricultura y la fauna acuática (Brack y Mendiola, 2004).

La contaminación de agua se debe a la liberación de contaminantes tóxicos contenidos en los residuos mineros y desde las obras mineras, los tajos abiertos, los socavones entre otros. Existen diferentes fuentes y mecanismos de liberación de estos contaminantes, el potencial de liberación de estos elementos y el riesgo asociado depende de las condiciones específicas del sitio, incluye el diseño, la operación de la extracción, del procesamiento de la extracción, gestión de residuos, calidad de medidas de mitigación, aspectos ambientales como el clima y la cercanía de los posibles receptores (Red Muqui, 2015).

La contaminación de suelo, se da por la remoción incontrolada de la capa vegetal y deforestación, en la fase extractiva de la explotación; puede abarcar considerables extensiones. Los procesos erosivos incontrolados producen cambios en el paisaje, asimismo, alteración en el drenaje, absorción de contaminantes transportados por vía aérea y luego depositados en el suelo (Brack y Mendiola, 2004).

En la contaminación del aire seda por la dispersión de sólidos en suspensión emitidos en cualquiera de las etapas de producción y el cierre. Así como los humos tóxicos que aniquilan la vegetación, afectan a la agricultura y la salud humana (Brack y Mendiola, 2004).

El impacto en la salud humana seda por las patologías respiratorias, oftalmológicas y problemas nerviosos (stress) en la población circundante, generados por ruidos, vibraciones, polvo y cambios del paisaje (Brack y Mendiola, 2004).

El impacto socioeconómico poder ser tanto directa como indirectamente por los proyectos mineros. Se puede incluir a las diferentes etnias y comunidades nativas compitiendo por recursos ambientales, es decir, la tierra y el agua que pueden ser reducidos en cantidad como resultado del 
proyecto minero (Brack y Mendiola, 2004).

El objetivo del trabajo de investigación fue evaluar el riesgo ambiental ocasionado por la presencia de pasivos mineros en la calidad del agua superficial de la localidad de San Miguel de Viso, distrito de San Mateo de Huánchor, provincia de Huarochirí, región de Lima, siendo los objetivos específicos, la identificación los PAMs, el diagnóstico de la calidad del agua superficial, la estimación del nivel de riesgo ambiental por la presencia de pasivos mineros, la priorización de los pasivos ambientales mineros que generen mayor riesgo en la calidad de agua superficial.

\section{Materiales y métodos}

Los materiales que se utilizaron Materiales fueron los que indica el protocolo nacional para el monitoreo de la calidad de los recursos hídricos superficiales aprobado mediante R.J. $\mathrm{N}^{\circ}$ 010-2016-ANA; mapas cartográficos donde se utilizó el programa ArcMap, del área de estudio, la ficha de identificación de PAMs y la guía de evaluación de riesgo del MINAM.

Antes de establecer el nivel de riesgo ambiental, primero se estableció la ubicación del área de estudio (localidad de San Miguel de Viso, distrito de San Mateo de Huánchor, provincia de Huarochirí). Luego, se procedió a preparar la evaluación preliminar, esta etapa consistió en la recopilación análisis de la mayor cantidad de información disponible referente al área de estudio en los entornos humano, natural y socioeconómico, dicha información permitió diseñar un mapa base preliminar del área de estudio donde se ubicó los PAMs existentes según el inventario MINEM.

Se realizó visitas al área de estudio en dos ocasiones; durante la primera visita campo se confirmó la existencia de los PAMs declarados en el inventario del Ministerio de Energía y Minas, en caso de identificar PAMs que no se encuentren en dicho inventario, se requirió de una ficha de campo para su identificación, la misma que se elaboró según las fichas de campo PERCAN, también se validó y actualizó de la información obtenida en gabinete. Para fines del presente trabajo de investigación solo se evaluó la contaminación al agua superficial como único componente ambiental afectado, para cada uno de los PAMs que estén presentes en el área de estudio y se realizó diagnóstico de la calidad de agua. En el segundo viaje se realizó la toma de muestra de agua de los PAMs que generen drenaje.

\section{La estimación del riesgo ambiental}

Se basó en la guía de evaluación de riesgo ambiental y la identificación de peligro, son todos los PAMs que generan drenaje, la identificación se realizó en campo y con ayuda de una ficha de campo (Figura 1).

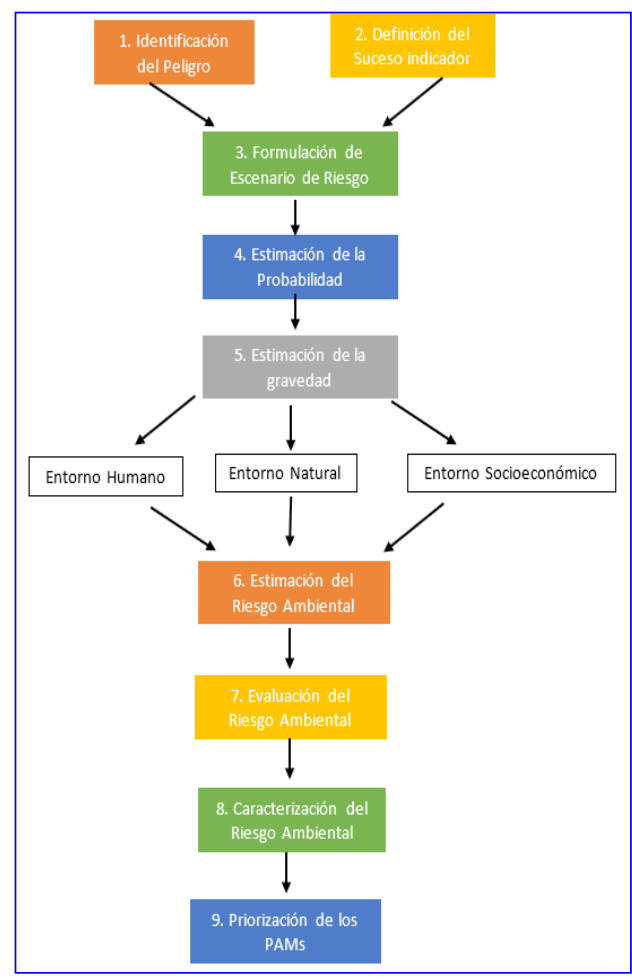

Figura 1. Metodología de estimación de riesgo ambiental 
Definición del suceso indicador, en esta etapa se estableció variables medibles que están determinados por los parámetros del diagnóstico de la calidad ambiental. Los parámetros analizados son todos aquellos que caracterizan a un PAM que presenta drenaje tales como ( $\mathrm{pH}$, metales, sulfatos, e entre otros).

Formulación de escenario, en esta etapa se estableció una serie de eventos, que origina potenciales consecuencias de contaminación a los tres entornos (humano, natural y socioeconómico) para cada suceso indicador

Estimación de la probabilidad, para poder determinar la probabilidad se asignó una escala de valoración que recomienda la guía de evaluación de riesgo, teniendo en cuneta el nivel de ocurrencia de la generación de drenaje que generan los PAMs si estos son continuos, intermitentes, y/o eventuales.

Estimación de la gravedad, para determinar esta variable se aplicó en forma diferenciada para cada entorno, natural, humano $\mathrm{y}$ socioeconómico conforme, siguiendo la siguiente formula.

En el entorno humano se determinó en función de la afectación a la salud de la población.

$$
\text { Salud }=C+2(P)+E_{h}+P b l
$$

Donde: C, cantidad de concentración del contaminante. $\mathbf{P}$, peligrosidad referido a la persona. Eh, extensión en el entorno humano, distancia en $(\mathrm{km})$ del PAM al centro poblado. $\mathbf{P b l}$, población, número de habitantes en el centro poblado.

Entorno natural se determinó en función de la afectación de la calidad del ambiente:

\section{Calidad del amb $=C+2(P)+E_{n}+C M$}

C, cantidad de concentración del contaminante. $\mathbf{P}$, peligrosidad referido al medio ambiente. Eh, Extensión en el entorno natural, distancia en $(\mathrm{km})$ de influencia del impacto. CM, Calidad del medio, número de indicadores afectados en el componente agua que superan el ECA de agua Categoría A2.

En el entorno socioeconómico se determinaron en función de información recabada en campo.

$$
\text { Socioeconómico }=C+2(P)+E_{h}+P C P
$$

C, cantidad, número de conflictos socio ambientales, número de fuentes de agua contaminada por PAM para el riego de agricultura. P, peligrosidad, fase en la cual se encuentra el conflicto socioambiental, número de fuentes de agua que existe para el desarrollo de su agricultura. Eh, extensión en el entorno humano, tiene el mismo valor que el En. PCP, patrimonio capital productivo, cantidad en porcentaje de parámetros que superan el ECA.

Estimación del riesgo ambiental: esta variable es el producto de la probabilidad por la gravedad de las consecuencias evaluado para cada escenario de riesgo en cada entorno (Figura 2).

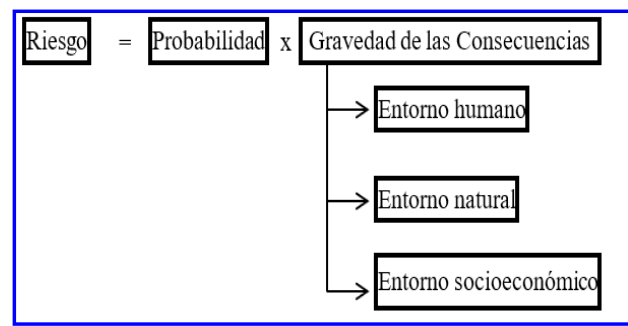

Figura 2. Estimación del riesgo ambiental 
Evaluación del riesgo ambiental: en esta etapa se interpretó el valor de estimación de riesgo determinado anteriormente, en una matriz de doble entrada según la escala de significación de riesgo en leve, moderado y significativo (Figura 3 y 4 ).

\section{Caracterización del riesgo ambiental:} en esta etapa se determinó el nivel riesgo ambiental para el PAM.

$$
C R_{P A M S}=(E H+E N+E S) / 3
$$

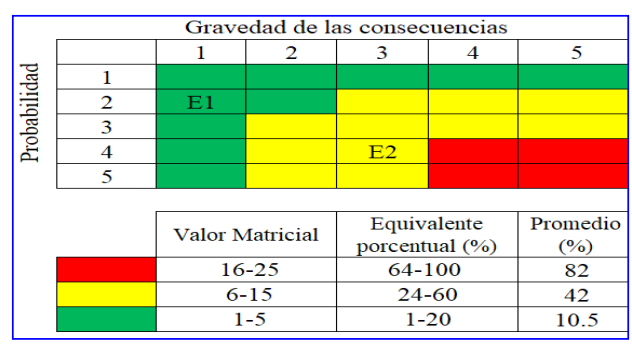

Figura 3. Matriz del riesgo ambiental

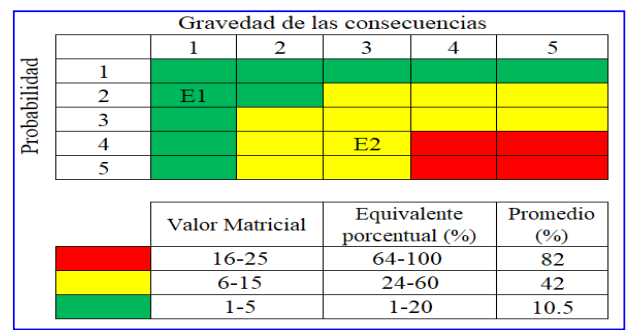

Figura 4. Escala de significancia de riesgo

CRPAMs, caracterización del Riesgo del PAM. EH, riesgo equivalente en el entorno humano. EN, riesgo equivalente en el entorno Natural. ES, riesgo equivalente en el entorno. socioeconómico.

Priorización de los PAMs: en esta etapa se prioriza los valores obtenidos de porcentaje de la caracterización de riesgo ambiental, en función de la escala de significancia de riesgo (Figura 4).

\section{Resultados y discusión}

\section{Identificación de PAM}

Para la identificación de los PAM se usó una ficha de campo identificación, donde se identificó 20 PAM de los cuales cuatro de ellos presentan drenaje, los mismos que se analizaron y se evaluaron para determinar el nivel de riesgo en la calidad de agua superficial. Asimismo, indicar que de los 20 PAM identificados dentro del área de estudio, 09 de ellos no se encontraban registrados dentro del inventario de pasivos del MINEM, estos pasivos son el PAS-1, PAS-12, PAS-13, PAS-15, PAS-16, PAS-17, PAS-18, PAS-19 y PAS-20.

\section{Diagnóstico de la calidad de agua superficial}

Para determinar el diagnostico se estableció cuatro puntos de monitoreo de los PAMs que generan drenaje, escorrentía y lixiviado, siguiendo el protocolo nacional para el monitoreo de la calidad de los recursos hídricos superficiales de la Autoridad Nacional del Agua (ANA) (Tabla 2).

Los parámetros que se analizaron insitu fueron: temperatura, $\mathrm{pH}$, conductividad eléctrica. Los parámetros analizados en laboratorio por la empresa SGS del Perú: solidos disueltos totales, arsénico total, cadmio total, cobre total, hierro total, manganeso tol, plomo total, mercurio total, zinc total, sulfato.

Para determinar el nivel de riesgo que generan los PAMs en la calidad de agua superficial se hizo mediante la comparación de los parámetros con los Estándares Nacionales de Calidad Ambiental de la categoría 1-A2, conforme la resolución Jefatural $N^{\circ}$ 56-2018-ANA, que clasifica con el código de curso 1375545 perteneciente a la cuenca del Rímac (Tabla 3). 
Tabla 1. Identificación de PAM

\begin{tabular}{|c|c|c|c|c|c|c|c|}
\hline \multirow{2}{*}{$\mathbf{N}^{\circ}$} & \multirow{2}{*}{$\begin{array}{c}\text { Código } \\
\text { PAM }\end{array}$} & \multirow{2}{*}{ Tipo de Pasivo } & \multicolumn{2}{|c|}{ Coordenadas } & \multirow{2}{*}{ Zona } & \multirow{2}{*}{$\begin{array}{c}\text { Distancia } \\
\text { al Centro } \\
\text { Poblado }\end{array}$} & \multirow{2}{*}{ Comentarios } \\
\hline & & & Norte & Este & & & \\
\hline 1 & PAS-1 & Tolva de $\mathrm{m}$ & 8694527 & 354470 & $18 \mathrm{~S}$ & $1,20 \mathrm{~km}$ & A 50 metros de la quebrada de Viso \\
\hline 2 & PAS-2 & Bocamina & 8694473 & 354541 & $18 \mathrm{~S}$ & $1,11 \mathrm{~km}$ & No hay presencia de cuerpos receptores \\
\hline 3 & PAS-3 & Taller & 8694489 & 354554 & $18 \mathrm{~S}$ & $1,10 \mathrm{~km}$ & No hay presencia de cuerpos receptores \\
\hline 4 & PAS-4 & Desmonte de mina & 8694238 & 356285 & $18 \mathrm{~S}$ & $0,69 \mathrm{~km}$ & No hay presencia de cuerpos receptores \\
\hline 5 & PAS-5 & Desmonte de mina & 8694113 & 356396 & $18 \mathrm{~S}$ & $0.84 \mathrm{~km}$ & No hay presencia de cuerpos receptores \\
\hline 6 & PAS-6 & Infraestructura & 8693916 & 356714 & $18 \mathrm{~S}$ & $1,22 \mathrm{~km}$ & No hay presencia de cuerpos receptores \\
\hline 7 & PAS-7 & Campamento & 8693774 & 356808 & $18 \mathrm{~S}$ & $1,37 \mathrm{~km}$ & No hay presencia de cuerpos receptores \\
\hline 8 & PAS-8 & Relavera & 8693841 & 356789 & $18 \mathrm{~S}$ & $1,32 \mathrm{~km}$ & $\begin{array}{l}\text { Hay } \\
\text { empo }\end{array}$ \\
\hline 9 & PAS-9 & Campamento & 8693747 & 356837 & $18 \mathrm{~S}$ & $1,41 \mathrm{~km}$ & No hay presencia de cuerpos receptores \\
\hline 10 & PAS-10 & Rampa & 8693756 & 356864 & $18 \mathrm{~S}$ & $1,43 \mathrm{~km}$ & No hay presencia de cuerpos receptores \\
\hline 11 & & $\begin{array}{l}\text { Plante } \\
\text { Proce }\end{array}$ & 869 & 35 & $18 \mathrm{~S}$ & $\mathrm{~km}$ & $\mathrm{~N}$ \\
\hline 12 & PAS-12 & Tolva de mineral & 8693734 & 356849 & $18 \mathrm{~S}$ & $1,43 \mathrm{~km}$ & No hay presencia de cuerpos receptores \\
\hline 13 & PAS-13 & Tolva de mineral & 8693745 & 356886 & $18 \mathrm{~S}$ & $1,45 \mathrm{~km}$ & Presencia de cuerpo receptor a 4 metros \\
\hline 14 & PAS-14 & Bocamina & 8693744 & 356935 & & $1,50 \mathrm{~km}$ & Presencia de cuerpo receptor a 4 metros \\
\hline 15 & PAS-15 & Infraestructura & 8693766 & 357078 & $18 \mathrm{~S}$ & $1,61 \mathrm{~km}$ & No hay presencia de cuerpos receptores \\
\hline 16 & PAS-16 & Bocamina & 8693963 & 357116 & $18 \mathrm{~S}$ & $1,56 \mathrm{~km}$ & No hay presencia de cuerpos receptores \\
\hline 17 & PAS-17 & Chatarra & 8693972 & 357095 & $18 \mathrm{~S}$ & $1,54 \mathrm{~km}$ & No hay presencia de cuerpos receptores \\
\hline 18 & PAS-18 & Relavera & 8694190 & 357157 & $18 \mathrm{~S}$ & $1,54 \mathrm{~km}$ & No hay presencia de cuerpos receptores \\
\hline 19 & PAS-19 & Campamento & 8694103 & 357094 & $18 \mathrm{~S}$ & $1,50 \mathrm{~km}$ & No hay presencia de cuerpos receptores \\
\hline 20 & PAS-20 & Bocamina & 8694185 & 356301 & $18 \mathrm{~S}$ & $0,72 \mathrm{~km}$ & No hay presencia de cuerpos receptores \\
\hline
\end{tabular}

En la Tabla 3 se puede observar que para el punto PAS-1 el $43 \%$ de los parámetros superan el ECA, para el PAS-8 el 93\% de los parámetros su superan el ECA, para el PAS-14 el 57\% de los parámetros superan el ECA, para el PAS-16 el $86 \%$ de los parámetros superan el ECA.

\section{Estimación del riesgo ambiental}

En las Figuras 5, 6 y 7, donde se muestra el grado de significancia del riesgo obtenido de los escenarios de riesgo del entorno humano, natural y socioeconómico para el PAS1, respectivamente. Donde la generación de aguas con variación significativa de temperatura representa un riesgo leve y aquellas con contenido de sólidos disueltos totales, $\mathrm{pH}, \quad$ conductividad eléctrica, sulfatos, aluminio, arsénico, cobre, hierro, mercurio y plomo representan un riesgo moderado para el entorno humano; mientras que la generación de aguas con contenido de cadmio, manganeso y zinc representan un riesgo significativo (Figuras 5). Además, las aguas con contenido de aluminio, arsénico, cobre, hierro, mercurio, plomo, temperatura y $\mathrm{pH}$ representan un riesgo moderado para el entorno natural; mientras que la generación de aguas con contenido de sólidos disueltos totales, conductividad eléctrica, sulfato, manganeso y zinc representan un riesgo significativo (Figuras 6). En los conflictos socio ambientales y el riego de áreas agrícolas con aguas contaminadas representan un riesgo moderado para el entorno socioeconómico (Figuras 7). 
Tabla 2. Identificación de puntos de monitoreo de agua superficial

\begin{tabular}{ccrl}
\hline \multirow{2}{*}{ Punto } & \multicolumn{2}{c}{ Coordenadas UTM } & \\
& Este & Norte & Descripción \\
\hline PAS-1 & 8694527 & 354470 & $\begin{array}{l}\text { Tolva de } \\
\text { mineral }\end{array}$ \\
PAS-8 & 8693841 & 356789 & Relavera \\
PAS-14 & 8693744 & 356935 & Bocamina \\
PAS-16 & 8693963 & 357116 & Bocamina \\
\hline
\end{tabular}

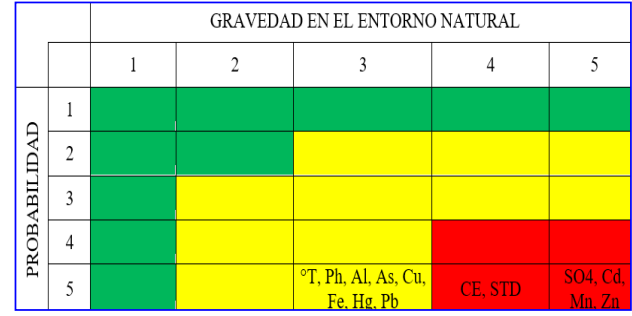

Figura 6. Riesgo ambiental del entorno natural para el PAS-1

Tabla 3. Resultados de la calidad de agua

\begin{tabular}{|c|c|c|c|c|c|c|}
\hline Parámetros & Unidad & PAS-1 & PAS-8 & PAS-14 & PAS-16 & ECA \\
\hline Temperatura & ${ }^{\circ} \mathrm{c}$ & 14 & 6,8 & 6,7 & 6,3 & $<8,22-14,22>$ \\
\hline $\mathrm{pH}$ & $\mathrm{pH}$ & 7,3 & 2,24 & 5,42 & 2,32 & $<5,5-9-0>$ \\
\hline Conductividad Eléctrica & $\mathrm{uS} / \mathrm{cm}$ & 1840 & 5450 & 1534 & 5820 & 1600 \\
\hline Sulfatos & $\mathrm{mg} / \mathrm{L}$ & 1385,31 & 2404,65 & 1002,56 & 2113,85 & 500 \\
\hline Sólidos Disueltos Totales & $\mathrm{mg} / \mathrm{L}$ & 1826 & 5064 & 1452 & 6040 & 1000 \\
\hline Aluminio Total & $\mathrm{mg} / \mathrm{L}$ & 0,109 & 65,356 & 1,282 & 22,283 & 5 \\
\hline Arsénico Total & $\mathrm{mg} / \mathrm{L}$ & 0,0024 & 151,39722 & 0,10512 & 121,43509 & 0,01 \\
\hline Cadmio Total & $\mathrm{mg} / \mathrm{L}$ & 0,06209 & 1,13539 & 0,07756 & 2,17927 & 0,005 \\
\hline Cobre Total & $\mathrm{mg} / \mathrm{L}$ & 0,07161 & 20,00241 & 0,70445 & 29,88764 & 2 \\
\hline Hierro Total & $\mathrm{mg} / \mathrm{L}$ & 0,9946 & 1481,3644 & 2,8783 & 745.9291 & 1 \\
\hline Manganeso Total & $\mathrm{mg} / \mathrm{L}$ & 5,11521 & 12.558 & 5,35218 & 16,77209 & 0,4 \\
\hline Mercurio Total & $\mathrm{mg} / \mathrm{L}$ & $<0,00009$ & 0,01125 & $<0,00009$ & $<0,00009$ & 0,002 \\
\hline Plomo Total & $\mathrm{mg} / \mathrm{L}$ & 0,0014 & 800,030 & 0,2174 & 2,7196 & 0,05 \\
\hline Zinc Total & $\mathrm{mg} / \mathrm{L}$ & 16,2195 & 260,3914 & $<0,00045$ & 493,9288 & 5 \\
\hline $\begin{array}{l}\text { Porcentaje de parámetros } \\
\text { que superan el ECA }\end{array}$ & & $43 \%$ & $93 \%$ & $57 \%$ & $86 \%$ & \\
\hline
\end{tabular}

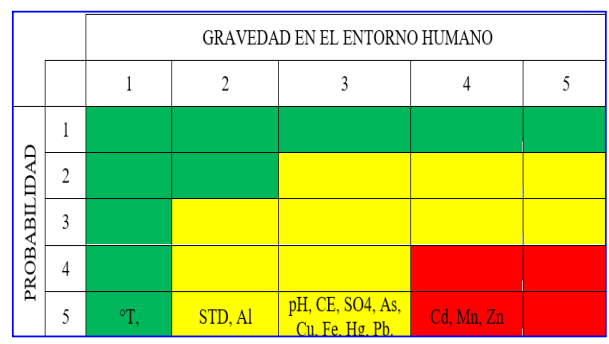

Figura 5. Riesgo ambiental del entorno humano para el PAS-1

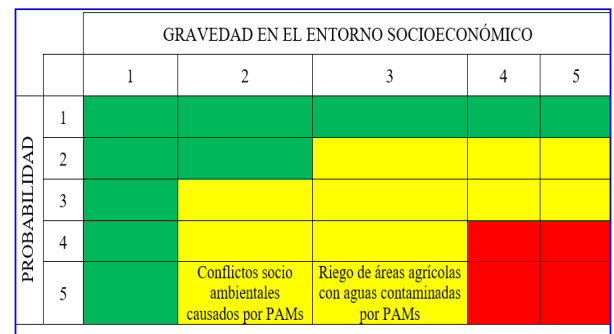

Figura 7. Riesgo ambiental del entorno socioeconómico para el PAS-1 
Por otra parte, también se presentan las Figuras 8, 9 y 10, donde se muestra el grado de significancia del riesgo obtenido de los escenarios de riesgo del entorno humano, natural y socioeconómico para el PAS8, respectivamente. Donde la generación de aguas con variación significativa de temperatura representa un riesgo leve y aquellas con contenido de sólidos disueltos totales, $\mathrm{pH}$, y sulfatos representan un riesgo moderado para el entorno humano; mientras que la generación de aguas con contenido de conductividad eléctrica, aluminio, arsénico, cadmio, cobre, hierro, manganeso, mercurio, plomo y zinc representan un riesgo significativo (Figura 8). Además, la generación de aguas con variación significativa de temperatura representa un riesgo moderado para el entorno natural; mientras que la generación de aguas con contenido de $\mathrm{pH}$, sólidos disueltos totales, conductividad eléctrica, sulfato, aluminio, arsénico, cadmio, cobre, hierro, manganeso, mercurio, plomo y zinc representan un riesgo significativo (Figura 9). Finalmente, los conflictos socio ambientales y el riego de áreas agrícolas con aguas contaminadas representan un riesgo moderado para el entorno socioeconómico (Figura 10).

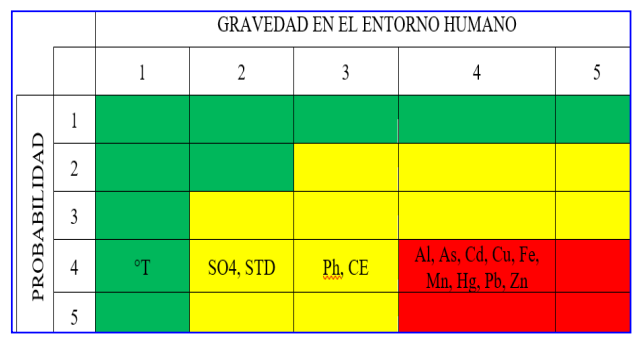

Figura 8. Riesgo ambiental del entorno humano para el PAS-8

En las Figuras 11, 12 y 13, donde se muestra el grado de significancia del riesgo, entorno humano, natural y socioeconómico para el PAS-14, respectivamente. Donde la generación de aguas con variación significativa de temperatura representa un riesgo leve y aquellas con contenido de sólidos disueltos totales, $\mathrm{pH}$, conductividad eléctrica, sulfatos, aluminio, cobre, mercurio $\mathrm{y}$ zinc representan un riesgo moderado para el entorno humano; mientras que la generación de aguas con contenido de arsénico, cadmio, hierro, manganeso y plomo representan un riesgo significativo (Figura 11). Además, la generación de aguas con variación significativa de temperatura, conductividad eléctrica, con contenido de aluminio, cubre, mercurio y zinc representan un riesgo moderado para el entorno natural; mientras que la generación de aguas con contenido de $\mathrm{pH}$, sólidos disueltos totales, sulfato, arsénico, cadmio, hierro, manganeso y plomo representan un riesgo significativo (Figura 14). Finalmente, los conflictos socio ambientales y el riego de áreas agrícolas con aguas contaminadas representan un riesgo moderado para el entorno socioeconómico (Figura 13).

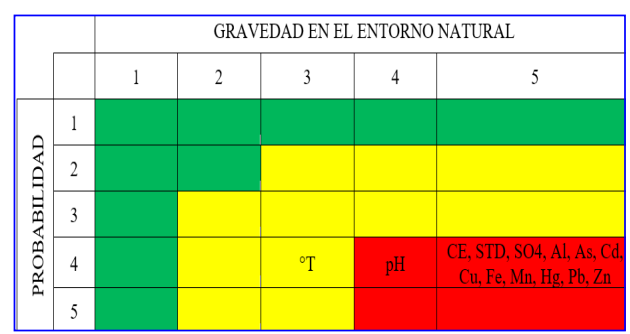

Figura 9. Riesgo ambiental del entorno natural para el PAS-8

En las Figura 14, 15 y 16, donde se muestra el grado de significancia del riesgo, entorno humano, natural y socioeconómico para el PAS-16, respectivamente. Donde la generación de aguas con variación 
significativa de temperatura representa un riesgo leve y aquellas con contenido de sulfatos, sólidos disueltos totales, $\mathrm{pH}$ y mercurio representan un riesgo moderado para el entorno humano; mientras que la generación de aguas con variación de conductividad eléctrica y con contenido de aluminio, arsénico, cadmio, cobre, hierro, manganeso, plomo y zinc representan un riesgo significativo (Figura 14). Además, la generación de aguas con variación de temperatura y con contenido de mercurio representan un riesgo moderado para el entorno natural; mientras que la generación de aguas con variaciones de $\mathrm{pH}$ y conductividad eléctrica, con contenido de sólidos disueltos totales, sulfato, aluminio, arsénico, cadmio, cobre, hierro, manganeso, plomo y zinc representan un riesgo significativo (Figura 15). Y en los conflictos socioambientales y el riego de áreas agrícolas con aguas contaminadas representan un riesgo moderado para el entorno socioeconómico (Figura 16).

\section{Caracterización del riesgo ambiental}

Para la priorización de lo PAMs se utilizó los valores de porcentaje obtenido de la evaluación de riesgo ambiental para cada pasivo conforme con las Tablas 4, 5, 6 y 7; la misma que se compara con la escala de significancia de riesgo de la Figura 4 para identificar en nivel de riesgo. Por tanto, en la Tablas 8 se muestra que la tolva de mineral (PAS-1) y la bocamina (PAS-14) representan un riesgo moderado para la calidad de agua superficial. Asimismo, la relavera (PAS-8) y la bocamina (PAS-16) representan un riesgo significativo a la calidad de agua superficial; y por tanto, estos dos últimos pasivos deben ser considerados como de atención prioritaria para su remediación.

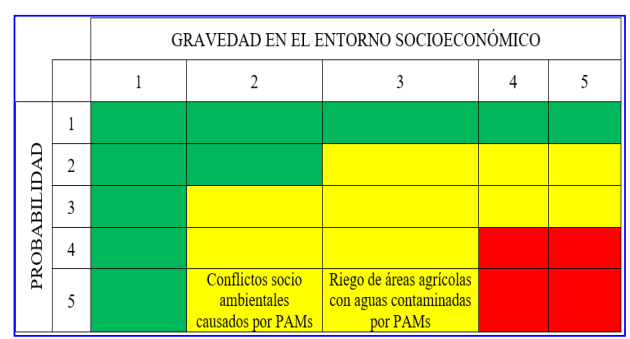

Figura 10. Riesgo ambiental del entorno socioeconómico para el PAS-8

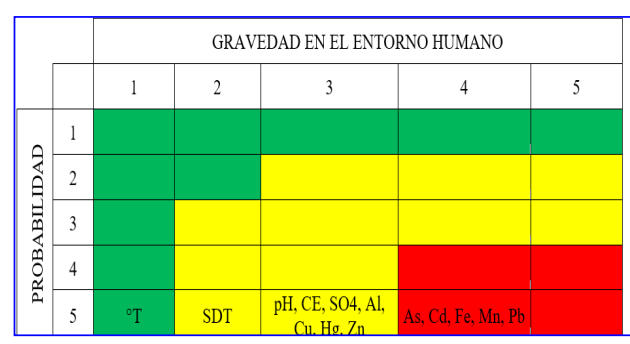

Figura 11. Riesgo ambiental del entorno humano para el PAS-14

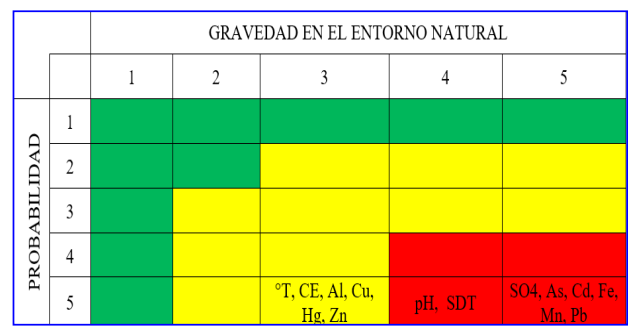

Figura 12. Riesgo ambiental del entorno natural para el PAS-14

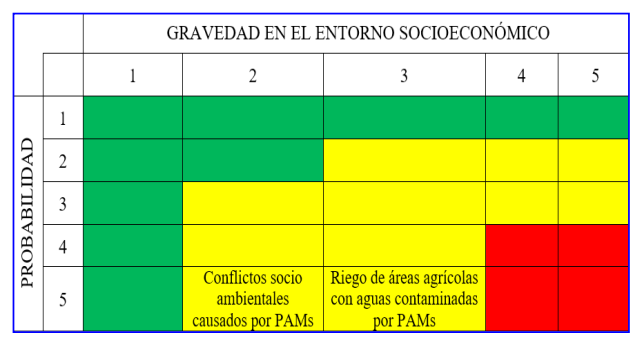

Figura 13. Riesgo ambiental del entorno socioeconómico para el PAS-14 


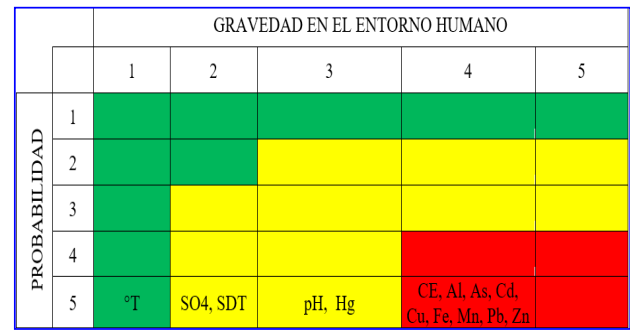

Figura 14. Riesgo ambiental del entorno humano para el PAS-16

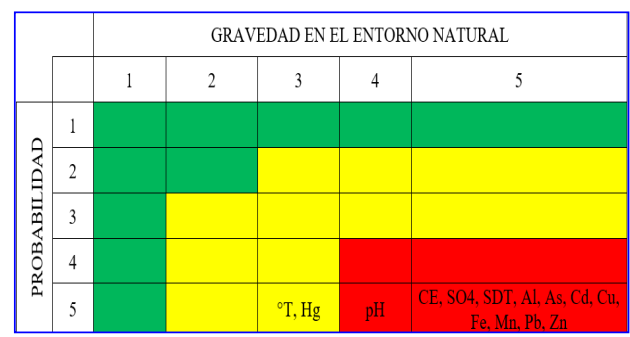

Figura 15. Riesgo ambiental del entorno natural para el PAS-16

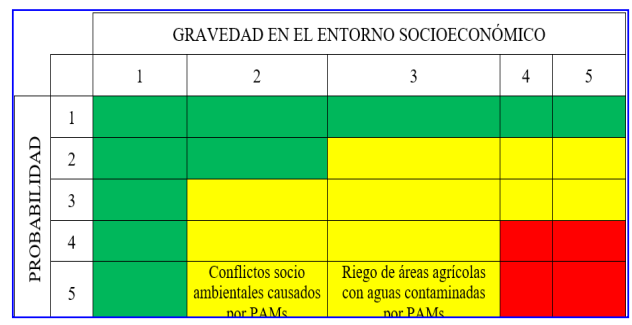

Figura 16. Riesgo ambiental del entorno socioeconómico para el PAS-16

Tabla 14. Riesgo ambiental para el PAS-1

\begin{tabular}{ccccc}
\hline Pasivo & Entorno & \multicolumn{4}{c}{$\begin{array}{c}\text { Equivalente Promedio } \\
(\%)\end{array}$} & $\begin{array}{c}\text { Riesgo } \\
\text { \% del } \\
\text { pasivo }\end{array}$ \\
\hline \multirow{4}{*}{ PAS-1 } & Humano & 48,32 & 42 & \\
& Natural & 59,14 & 42 & 42 \\
& $\begin{array}{c}\text { Socio- } \\
\text { económico }\end{array}$ & 42 & 42 & \\
\hline
\end{tabular}

Tabla 5. Riesgo ambiental para el PAS-8

\begin{tabular}{|c|c|c|c|c|}
\hline Pasivo & Entorno & $\begin{array}{c}\text { Equivalente } \\
(\%)\end{array}$ & $\begin{array}{l}\text { Promedio } \\
(\%)\end{array}$ & $\begin{array}{l}\text { Riesgo } \\
\% \text { del } \\
\text { pasivo }\end{array}$ \\
\hline \multirow{3}{*}{ PAS-8 } & Humano & 68,32 & 82 & \multirow{3}{*}{69} \\
\hline & Natural & 79,14 & 82 & \\
\hline & $\begin{array}{l}\text { Socio- } \\
\text { económico }\end{array}$ & 42 & 42 & \\
\hline
\end{tabular}

Tabla 6. Riesgo ambiental para el PAS-14

\begin{tabular}{ccccc}
\hline Pasivo & Entorno & \multicolumn{4}{c}{$\begin{array}{c}\text { EquivalentePromedio } \\
(\%)\end{array}$} & $\begin{array}{c}\text { Riesgo } \\
\text { \% del } \\
\text { pasivo }\end{array}$ \\
\hline \multirow{2}{*}{ PAS-14 } & Humano & 54,04 & 42 & \\
$\begin{array}{c}\text { Natural } \\
\text { Socio- } \\
\text { económico }\end{array}$ & 67,71 & 82 & 55 \\
\hline
\end{tabular}

Tabla 7. Riesgo ambiental para el PAS-16

\begin{tabular}{ccccc}
\hline Pasivo & Entorno & $\begin{array}{c}\text { Equivalente Promedio } \\
(\%)\end{array}$ & $\begin{array}{c}\text { Riesgo } \\
\text { \% del } \\
\text { pasivo }\end{array}$ \\
\hline \multirow{2}{*}{ PAS-16 } & Humano & 65,46 & 82 & \\
& $\begin{array}{c}\text { Natural } \\
\text { Socio- } \\
\text { económico }\end{array}$ & 76,29 & 82 & 69 \\
\hline
\end{tabular}

Tabla 8. Nivel de riesgo ambiental

\begin{tabular}{lccc}
\hline \multicolumn{1}{c}{ Pasivos } & ID & $\begin{array}{c}\text { Riesgo \% } \\
\text { del pasivo }\end{array}$ & $\begin{array}{c}\text { Nivel de } \\
\text { Riesgo }\end{array}$ \\
\hline $\begin{array}{l}\text { Tolva de } \\
\text { mineral }\end{array}$ & PAS-1 & 42 & Moderado \\
Relavera & PAS-8 & 69 & Significativo \\
Bocamina & PAS-14 & 55 & Moderado \\
Bocamina & PAS-16 & 69 & Significativo \\
\hline
\end{tabular}

\section{Conclusiones}

La microcuenca de Viso presenta un 46 pasivos registrados en el inventario del MEM. Sin embargo, en el área de estudio se identificó 20 PAMs, de los cuales nueve de ellos no se encuentran registrados en el inventario. Mientras que, los cuatro PAM que presentaron drenajes, se definieron 14 
parámetros a monitorear y con respecto al ECA de agua categoría 1- A2, del PAS1, PAS-8, PAS-14 y PAS-16 el 43\%, 93\%, $57 \%$ y $86 \%$ de los parámetros evaluados superaron el ECA, respectivamente. Además, el S04, SDT, Cd y Mn sobrepasan el ECA en los cuatro puntos de monitoreo.

El nivel de riesgo ambiental para el PAS1 en el entorno humano 48,32\%, natural $59,14 \%$ y socioeconómico $42 \%$, para el PAS-8 en el entorno humano 68,32\%, natural $79,14 \%$ y socioeconómico $42 \%$, para el PAS-14 en el entorno humano $54,04 \%$, natural $67,71 \%$ y socioeconómico $42 \%$ y para el PAS- 16 en el entorno humano $65,46 \%$, natural $76,29 \%$ y socioeconómico $42 \%$.

La metodología de evaluación de riesgo del MINAM, es una herramienta que usa criterios cualitativos y cuantitativos a través de indicadores medibles; a diferencia de la metodología que actualmente usa el MINEM que son las fichas PERCAN para la evaluación y priorización que se basa en criterios cualitativos. Los criterios de valoración para el entorno socioeconómico son mucho más dinámicos, a diferencia del entorno humano y natural que están más relacionados al diagnóstico de la calidad de agua. Dichos criterios se establecieron en función de la realidad de San Miguel de Viso y los escenarios de riesgo propuestos son conflictos socio ambientales y afectación al riego para la agricultura.

\section{Literatura citada}

Aranda A. 2013. Pasivos ambientales mineros en el Perú. Lima, Perú. 3pp.

Aramburo M. 2011. Requerimientos para el diseño de una metodología que permita estimar el valor pasivo ambientales mineros. Tesis de grado para optar al título de maestría. Universidad Nacional de Colombia.

ANA [Autoridad Nacional del Agua]. 2018
R.J. N056-2018-ANA. Aprobación

de la clasificación de los cuerpos de agua continentales superficiales.

ANA [Autoridad Nacional del Agua]. 2016 R.J. N010-2016-ANA. Aprobación

del protocolo nacional para el monitoreo de la calidad de agua de los recursos hídricos superficiales.

Barreño, C. 2018. Evaluación de los riesgos generados por pasivos ambientales en la minería de carbón, con enfoque de ecología política: estudio de caso municipio de Rondon (Boyacá). Bogotá, Colombia, Universidad Distrital Francisco José de Caldas. 24-30pp.

Brack y Mendiola. 2004. Ecología del PerúBruño.

Castro, V. 2013. Análisis de la percepción ambiental de la población de San

Miguel de Viso sobre la influencia de las actividades mineras en la calidad de agua. Lima, Perú, UNALM. 21-23, $45 \mathrm{pp}$.

Congreso de la Republica 2004. Ley marco del sistema nacional de gestión ambiental, aprobada mediante la Ley $\mathrm{N}^{\circ} 28245$.

Congreso de la Republica 2005. Modificatoria de los Artículos 5, 6, 7, 8, la primera disposición complementaria y final de la Ley $\mathrm{N}^{\circ} 28271$, ley que regula los pasivos ambientales en la actividad minera, y le añade una tercera disposición complementaria y final aprobada mediante Ley $\mathrm{N}^{\circ} 28526$.

Congreso de la Republica 2004. Regula los pasivos ambientales en la actividad minera, aprobado mediante la Ley $\mathrm{N}^{\circ}$ 28271.

Congreso de la Republica 2003. Ley que regula el cierre de minas, aprobada me. 
De la Puente, L. 2007. Regulación Ambiental Peruana y el ciclo de una mina. Lima, Perú. DELAPUENTE Abogados.

Defensoría del Pueblo. 2018. Reporte de conflictos sociales. Adjuntía para la prevención de conflictos sociales y la gobernabilidad. Lima, Perú. Reporte $\mathrm{N}^{\circ}$ 176. Disponible en: https://www. defensoria.gob.pe/documentos/ reporte-mensualde-conflictossociales-n-176/

DGAAM [Dirección General de Asuntos Ambientales Mineros]. 2013. MEM concluye remediación y cierre de 64 pasivos mineros en Cajamarca. Consultada el 18 de Septiembre del 2013. Disponible en: http://www. minem.gob.pe/_detallenoticia. php?idSector $=4 \&$ idTitular $=5684$.

DGAAM [Dirección General de Asuntos Ambientales Mineros]. 2010. Guía parala elaboración de planes de cierre de pasivos ambientales mineros.

DGM [Dirección General de minería]. 2009. Fichas de campo propuestas por el proyecto PERCAN para la identificación de pasivos ambientales mineros dentro de una ex unidad minera, aprobada mediante Resolución Directoral $\mathrm{N}^{\circ}$ 173-2009MEM-DGM.

FONAM [Fondo Nacional del Ambiente]. 2006. Inventario, diagnóstico y priorización de los pasivos ambientales en la cuenca del río Llaucano - Hualgayoc.

INGEMENT. [Instituto Geológico Minero y Metalúrgico]. 1983. Geología de los cuadrángulos de: Matucana $\mathrm{y}$ Huarochirí. Lima, Perú. Boletín $\mathrm{N}^{\circ}$ 36.

INGEMENT. [Instituto Geológico Minero y Metalúrgico]. 2005. Estudio de los recursos minerales del Perú, Franja $\mathrm{N}^{\circ}$ 4. Lima, Perú. Boletín $\mathrm{N}^{\circ} \mathrm{B} 14$.
Leturia J. 2008. Remediación de pasivos ambientales mineros $\mathrm{y}$ aprovechamiento económico. Revista peruana de derecho de la empresa. Perú 23-65-129-147pp.

María Ayora 2010. Análisis de aguas naturales y residuales. Universidad de Jaén- España.

MINAM [Ministerio del Ambiente]. 2010 Guía de evaluación de riesgo ambiental.

MINAM [Ministerio del Ambiente]. 2012. Glosario de términos para la formulación de proyectos ambientales.

MINAM [Ministerio del Ambiente]. 2012. Metodología para la estimación del nivel de riesgo de pasivos ambientales en el subsector hidrocarburos.

MINAM [Ministerio del Ambiente]. 2017. Estándares nacionales de calidad mbiental para agua, aprobado mediante D.S. N $^{\circ}$ 004-2017-MINAM. Lima, Perú.

MINEM [Ministerio de Energía y Minas]. 2005. Modifican reglamento de pasivos ambientales de la actividad minera aprobado por DS $\mathrm{N}^{\circ} 059$ 2005-EM mediante el DS $\mathrm{N}^{\circ} 003$ 2009EM. Ministerio de Energía y Minas. Lima, Perú.

MINEM [Ministerio de Energía y Minas]. 2005. Reglamento de pasivos ambientales de la actividad minera aprobado mediante el DS $\mathrm{N}^{\circ} 059$ 2005-EM. Ministerio de Energía y Minas. Lima, Perú.

MINEM [Ministerio de Energía y Minas]. 2010. R.M. $\mathrm{N}^{\circ}$ 129-2010-MEM/ DM Disponen que el Estado asuma la remediación de los pasivos ambientales mineros calificados de muy alto riesgo y alto riesgo ubicados en la región Cajamarca. 
MINEM [Ministerio de Energía y Minas]. 2013 R.M. N N $^{\circ}$ 094-2013-MEM/ DM. Encargan a la empresa estatal Activos Mineros S.A.C. ejecutar la remediación delos pasivos ambientales mineros de diversos proyectos ubicados en las regiones Áncash, Lima Cajamarca, Huancavelica, Pasco, Junín e Ica.

MINEM [Ministerio de Energía y Minas]. 2015 R.M. N N $^{\circ}$ 045-2015-MEM/ DM se dispone que el Estado asuma la remediación de 134 pasivos ambientales mineros de diversos proyectos de remediación ubicados en las regiones de Ancash, Lima, Puno y Cajamarca.

OIT [Organización Internacional de Trabajo]. 2012 enciclopedia de salud seguridad en el trabajo Capitulo 63 "Metales: Propiedades químicas y toxicas".

OMS [Organización Mundial de la Salud]. 2018 cuarta edición. "Guía para la calidad de agua de consumo humano".

RED MUQUI. 2010. Los Pasivos ambientales mineros: Diagnostico y propuesta. 11-12pp.

SERNAGEOMIN [Servicio Nacional de Geología y Minería]. 2008. Manual de evaluación de riesgos de faenas mineras abandonadas o paralizadas FMA/P - Chile.

SNMPE [Sociedad Nacional de Mineria Petroleo y Energia]. Diciembre. 2006. Informe $\mathrm{N}^{\circ} 41 \mathrm{El}$ ciclo productivo de la minería.

Sotomayor, A. 2016. Remediación de pasivos ambientales mineros como estrategia para el cuidado del ambiente (trabajo presentado en la Conferencia Académica Anual del Consorcio, octubre, 2015). En Consorcio de Universidades (Ed.), Metas del Perú al bicentenario (pp. 241-246). Lima: Consorcio de Universidades.

UNE 150008: 2008. Análisis y evaluación del riesgo ambiental.

Walter, M. 2009. Conflictos ambientales, socioambientales, ecológicos distributivos de contenido ambiental. Madrid, España. Pag 3 CIP-ECOSOCIAL (Centro de la investigación para la paz).

Yipari, A. 2003. Pasivos Ambientales Mineros en Sudamérica Informe elaborado para la Comisión Económica para América Latina y el Caribe - CEPAL, el Instituto Federal de Geociencias y Recursos Naturales - BGR, y el Servicio Nacional de Geología y Minería SERNAGEOMIN. 